Artículos / Articles

\title{
Experiencias y metáforas sobre la precariedad y la hiperactividad de la juventud en un tiempo de espera
}

\author{
Experiences and metaphors on precariousness and youth \\ hyperactivity in a time of waiting
}

\author{
Benjamín Tejerina (iD \\ Universidad del País Vasco-Euskal Herriko Unibertsitatea, España \\ b.tejerina@ehu.eus
}

Recibido / Received: 10/10/2019 Aceptado / Accepted: 03/11/2020

\section{RESUMEN}

La precariedad se entiende generalmente como una característica negativa, una ausencia o falta de competencias o recursos de algún tipo. Si la juventud se define como un tiempo de preparación anterior a la edad adulta, puede conducir a interpretar esta etapa del ciclo vital como un momento de dependencia, un precompleto. Ambas pueden entenderse también como espacio-tiempo de actividad, creatividad, y experimentación de nuevas estrategias de vida en condiciones determinadas. El artículo plantea tres objetivos: uno, sistematiza los estudios sobre precariedad realizados en España en los últimos años; dos, a partir de estas investigaciones presenta una definición compleja de precariedad. Basado en un trabajo de campo de entrevistas personales y grupo de discusión aborda, en tercer lugar, diferentes significados, narrativas y experiencias de los jóvenes sobre la normalidad y la precariedad, así como algunas metáforas comúnmente utilizadas para referirse a la precariedad.

Palabras clave: precariedad; juventud; metáforas; transición juvenil.

\begin{abstract}
Precarity is generally understood as a negative characteristic, an absence or lack of skills, competencies or resources of some kind. If youth is defined as a time of preparation prior to adulthood, it can lead to interpreting this stage of the life cycle as a moment of dependence, a pre-complete. Both can also be understood as a space-time of activity, creativity, and experimentation of new strategies of life in determined conditions. The article proposes three objectives: one, it systematizes the studies on precariousness carried out in Spain in recent years; two, from these investigations it presents a complex definition of precariousness. Based on a field work of personal interviews and discussion group, it addresses, in third place, different meanings, narratives and experiences of young people about normality and precariousness, as well as some metaphors commonly used to refer to precariousness.
\end{abstract}

Keywords: precarity; youth; metaphors; youth transition.

\footnotetext{
* Sugerencia de cita / Suggested citation: Tejerina, B. (2020). Juventud y crisis. Experiencias y metáforas sobre la precariedad y la hiperactividad de la juventud en un tiempo de espera. Revista Española de Sociología, 29 (3, supl. 2), 95-112.

https://doi.org/10.22325/fes/res.2020.76
} 


\section{LOS ANÁLISIS RECIENTES SOBRE LA PRECARIEDAD EN ESPAÑA}

La cuestión de la precariedad ha atraído la atención de numerosos investigadores en las últimas décadas, hasta el punto de convertir el concepto de precariado en una forma de definir las condiciones de vida de una parte significativa de la población en las sociedades contemporáneas (Standing, 2011). Una primera línea de investigación se ha centrado en la pérdida de centralidad del trabajo, y su repercusión en la identidad de las personas jóvenes (Santamaría, 2011). Otos trabajos han contribuido a la institucionalización de conceptos como empleo débil (Alonso, 2000), trabajadores flexibles y precarios (Bilbao, 1998; Díaz-Salazar, 2003; Zubero, 2006) o crisis del trabajo (Castel, 1997).

Una segunda línea de investigación sobre precariedad se ha centrado en los procesos de construcción de sujetos cuya característica más relevante es la carencia de algún atributo social, bien en términos identitarios bien en cuestiones materiales: las madres solteras (Tobío y Fernández Cordón, 1999; González, Jiménez y Morgado, 2004); la conciliación de la vida laboral, personal y familiar (Carrasquer y Torns, 2007; Prieto, Ramos y Callejo, 2008), la condición de migrante (Izaola, 2017) o los menores dependientes (Morente y Barroso, 2003).

La tercera línea de análisis se ha centrado en la juventud entendida como rito de paso (Van Gennep, 1986; Turner, 1988) y como identidad precaria, atendiendo a las condiciones salariales y la flexibilidad en el trabajo (Santos, 2003; Sánchez Moreno, 2004) que dificultan la emancipación juvenil y la realización de proyectos vitales, reforzando la relación de dependencia familiar (Rodríguez, 1999). Las situaciones que describen estas investigaciones son resultado de la limitación en los recursos necesarios para poder independizarse (Casal, 1996; López, 2005; Jurado, 2007). Según estos trabajos, las principales dificultades están relacionadas con el mercado de la vivienda (Echaves, 2018), la situación salarial, la temporalidad en el empleo y, también, el rol de la familia mediterránea, que condicionan las estrategias para alcanzar la vida adulta (Rico, 2005).

En cuarto lugar, se ha contemplado el problema de la inserción laboral que, bajo determinadas circunstancias, conduce a situaciones de exclusión social. Varios autores han llamado la atención sobre los efectos de las condiciones de trabajo para las nuevas generaciones en marcado contraste con las que vivieron las anteriores (Casal, García, Merino, Quesada, 2006; Jiménez et al., 2008).

Una quinta línea de investigación ha tenido en cuenta la dimensión política de la precariedad, entendida como la relación entre situación en el mercado laboral, procesos de emancipación y opción política que, aún habiéndose debilitado en las últimas décadas, continúa teniendo influencia (Parés y Subirats, 2016). Asimismo, se ha prestado una especial atención a la cultura política, planteada desde una visión que atiende a los comportamientos de la juventud, sobre todo a la relación que se establece entre orientación de voto y situación laboral, así como a la repercusión que tanto la ideología como la situación económica general tienen sobre esta relación (Salido y Martín, 2007).

En esta misma línea se sitúan trabajos relacionados con la cuestión del capital social, voluntariado, asociacionismo y movimientos sociales. Estos aspectos resultan importantes si tenemos en cuenta que desde el inicio de la crisis en 2008 se ha producido un notable incremento en la movilización en torno a la precarización social (Tejerina y Seguel, 2011; Portos y Masullo, 2017). Especial mención merecen los trabajos anteriores a la crisis de Montero, Font y Torcal (2006) sobre confianza política, capital social y asociacionismo, la relación entre capital social y rendimiento institucional (Subirats y Gallego, 2002) y el asociacionismo (Ariño, 2004).

En el contexto de la activación política se ha analizado la forma en que se relacionan la movilización contra la precariedad y el contexto de la estructura de oportunidad política 
(Pereda, 2002; Mosca, 2006; Della Porta, 2015). Algunas de las movilizaciones políticas más recientes en Túnez, Egipto o Marruecos, y, las del Movimiento 15-M en diversos lugares de España, han tenido un fuerte protagonismo de sectores juveniles (Ancelovici, Dufour y Nez, 2016; Tejerina y Perugorría, 2018).

En estos estudios se constata una gran diversidad de factores que impulsan la precariedad juvenil, así como múltiples efectos derivados de ella. Dos conclusiones claras emergen de estas investigaciones: en primer lugar, la precariedad es utilizada con distintos significados, pero en su mayoría vinculados con la carencia, ausencia o limitación de recursos; y, en segundo lugar, lejos de caracterizar un periodo de baja actividad, espolea la búsqueda de recursos y estrategias para salir de estas situaciones. Sin embargo, esta dimensión estratégica adolece de una adecuada teorización, aspecto que se aborda a continuación.

\section{EL CONCEPTO DE PRECARIEDAD: UNA AMPLIACIÓN DE SUS SIGNIFICADOS}

El término precarioำ, originalmente reservado a ámbitos como la salud, la edificación, la acción de gobierno o la paz, se suele encontrar en el lenguaje coloquial asociado a un determinado tipo de coyuntura, situación o estado de cosas caracterizado por la falta de solidez o de estabilidad. Se puede considerar como una condición cuando se asocia a algo permanente o como una situación duradera que se experimenta en un contexto de limitación para disponer de medios y recursos. Como ha señalado Le Blanc (2007), el sentido antropológico de la precariedad está asociado a la contingencia que caracteriza la condición humana.

El discurso sobre la precariedad ha estado presente en las ciencias sociales asociado a categorías como anomía, alienación, marginalidad o pobreza. Un elemento que aparece con regularidad en su desarrollo conceptual es el de inseguridad, un concepto que, en la época contemporánea, lo encontramos vinculado a los de riesgo, incertidumbre o complejidad.

Con regularidad la lucha contra la precariedad aparece como justificación de la expansión del Estado de bienestar y sus políticas de inserción (Castel, 1997, p. 324). En el ámbito francés y anglosajón, la expansión del término se produce primero en relación a la pobreza asociada a las familias vulnerables (Pitrou, 1978), después se vincula con el estatus del empleo (Schnapper y Villac, 1989) y en la década de 1990 en relación con la carencia de trabajo (Paugam, 1991; 2000).

Lo precario experimenta un desplazamiento semántico desde lo marginal a un progresivo acercamiento al campo del trabajo y del empleo, y, en autores como Touraine (1978), Giddens (1984), Bourdieu (1999) y Beck (2000), y su sentido se traslada a la estructura social o a los procesos estructurantes de las sociedades contemporáneas. Sin embargo, en ninguno de los casos analizados se plantea la hipótesis de una generalización de la precariedad más allá de sectores marginales, de colectivos concretos o de contextos que afectan a individuos en condiciones especiales.

Este artículo se inscribe dentro de la idea de la generalización de la precariedad en las sociedades contemporáneas, lo que significa que ha dejado de ser propio de minorías para convertirse en una experiencia común que afecta a crecientes sectores sociales, incluyendo a clases medias y sectores tradicionalmente considerados previlegiados. Esta hipótesis tiene importantes consecuencias a nivel metodológico que abordaremos más adelante.

1 Un desarrollo pormenorizado de las relaciones entre crisis y precariedad vital, así como una versión previa de las definiciones que se utilizan en este artículo pueden encontrarse en el texto de Tejerina, Cavia, Santamaría y Carbajo (2012). 
La precariedad es un concepto que abarca múltiples aspectos de la vida, pero su desarrollo reciente se ha producido a través de los procesos que giran en torno al mercado laboral y sus condicionamientos sobre la vida y las relaciones sociales de los sujetos (Sennet, 2000; Prieto, 2008).

La precariedad puede entenderse como una situación estructural o como un contexto coyuntural donde las personas se ven obligadas a actuar. Con este sentido se ha analizado fundamentalmente la precariedad que procede del ámbito laboral, pero que se extiende a otros ámbitos de la vida de las personas, aunque su consideración ha gozado de menor atención. La precariedad vital puede definirse como

\begin{abstract}
" aquella situación de origen estructural o coyuntural caracterizada por una restricción o limitación de acceso a las condiciones, requisitos y recursos considerados necesarios para poder planear, llevar a cabo y gestionar una vida autónoma. El nivel de restricción o limitación puede alcanzar diferentes grados de intensidad en relación con los recursos medios disponibles en una sociedad determinada. La precariedad es, pues, una categoría relacional en un doble sentido: a) en relación con la media de la sociedad, grupo o categoría social de que se trate; y b) en relación con los diversos ámbitos existenciales » (Tejerina et al., 2012, p. 22).
\end{abstract}

La precariedad es un concepto que aúna la condición y la situación personal en la relación del individuo con el medio. La precariedad es el estado al que se llega mediante procesos de precarización. Los procesos de precarización afectan a la identidad en la medida en que los individuos pierden o ven alteradas las modalidades de apego del yo al nosotros y a los tú.

La precarización (y la afluencia) como proceso toca diferentes dimensiones que tienen que ver con salidas o entradas en zonas de riesgo (y protección), y que hace referencia a la capacidad de gestión de recursos y competencias de los individuos: trabajo, remuneración, consumo, residencia, cualificación educativa, entorno, vida familiar y afectiva, relaciones sociales, salud y participación cívica. En la precarización como proceso también participan las instituciones, por medio de las prácticas de la acción pública o por su ausencia, y a través de la normativización de la precariedad, en la medida en que a través de programas sociales enseñan, orientan e impulsan a los individuos a moverse en la búsqueda de protección social.

Aunque en la mayor parte de los casos la experimentación de la precariedad, la gestión cotidiana de una existencia llena de posibles limitaciones, y las estrategias desarrolladas por individuos y colectivos, vienen cargadas de consecuencias sociales muy negativas, no eliminan la posibilidad de que también puedan entenderse y analizarse bajo el principio de la creatividad social, de la innovación, a partir de la búsqueda más o menos imaginativa de soluciones en una situación de restricción de recursos típica.

Considerada desde esta perspectiva, la precariedad no es únicamente una carencia puntual y limitante, una forma de estigma social, sino un dato estructural y generalizado, puesto que se ha convertido en algo inscrito en la vida social de numerosas personas. Si esta afirmación es cierta, y los datos empíricos disponibles apuntan en esta dirección, la precariedad se estaría desplazando desde los sectores marginales, próximos en muchos casos a la exclusión social, a posiciones más centrales de la estructura social (Llopis y Tejerina, 2016).

La precariedad no aparece ya como un fallo del sistema que hay que reparar (discurso de la exclusión/integración), como una forma más o menos transparente de conflicto social (Dahrendorf, 1990), sino que las situaciones precarias son un mecanismo asociado al propio funcionamiento social (Paugam, 2007; Mendiguren, García y Ruiz-Estramil, 2017).

Cabe plantear que este mecanismo se ha acelerado en lo que podemos denominar modernidad tardía y en el momento presente, instalándose en el centro mismo de la vida social. En definitiva, la precariedad ya no opera sólo como generador de espacios 
de exclusión, residuales, marginales, sino que se ha generalizado, y se ha convertido en un definidor de situaciones sociales concretas y cotidianas. $Y$, en el mismo sentido, en un concepto articulador de definiciones sociológicas que ya no pueden explicarse mediante el vocabulario clásico de las ciencias sociales (Tejerina et al., 2012, p. 23).

Los diversos procesos de precarización y sus múltiples formulaciones pueden ordenarse en torno a una distinción entre dos definiciones teórico-metodológicas: la de la precariedad simple y la de la precariedad generalizada o precariedad compleja.

La precariedad simple se entiende como sinónimo de carencia, en el sentido más pegado a la definición de uso frecuente. Es, pues, un rasgo negativo, algo puntual que ha de ser resuelto: carencia, falta, inestabilidad, inseguridad, insuficiencia. Esta forma de entender la precariedad se corresponde con situaciones de crisis normativa, que producen espacios sociales altamente vulnerables, carentes y desintegrados.

El segundo tipo de precariedad entiende la misma como tenencia, como posibilitador. La precariedad ya no es vista únicamente como algo a solucionar, sino que se define como mundo de vida (Schütz y Luckmann, 2001) que teniendo como dato su inestabilidad requiere gestionarse, y que obliga a desarrollar estrategias singulares. De esta manera, la precariedad no es una situación pasajera, sino que se convierte en una precariedad generalizada o precariedad compleja.

El interés de la precariedad compleja reside en que permite analizar mejor los significados de las experiencias vividas y la presencia de las distintas estrategias de gestión que desarrollan quienes se ven confrontados a la precariedad vital, dando lugar a nuevas formas de estar y pensar lo precario. La hipótesis que planteamos es que al ser la juventud uno de los sectores afectados por los procesos de precarización, los mundos de la precariedad están presentes de manera generalizada o se hacen presentes de manera más intensa en algunas situaciones de la vida social juvenil. Con ello no pretendemos afirmar que no existan diferencias significativas entre distintos sectores juveniles según niveles de estudio, edad, origen social, residencia o capital social. Estas segmentaciones continúan teniendo relevancia sociológica, pero lo que intentamos es ir más allá de ellas, para señalar que en las últimas décadas se ha producido una progresiva invasión de sectores sociales medios y profesionales, que tradicionalmente se consideraban a salvo de la precariedad, hasta generalizar las experiencias de la precariedad vital en sectores afluentes.

\section{PLANTEAMIENTO DE LA INVESTIGACIÓN Y METODOLOGÍA}

El trabajo de campo no se ha centrado en los sectores de la población que vienen experimentando un intenso proceso de precarización con anterioridad a la crisis financiera de 2008. Ni se ha acudido a sectores marginales o próximos a la exclusión social, donde parece evidente que la precariedad se manifiesta con mayor fuerza. Las personas entrevistadas son jóvenes emancipados o forman parte de familias que proceden de sectores medios, profesionales, con capital social y cultural medio-alto, que pueden disponer de recursos institucionales o contribuciones familiares de apoyo, y que en ningún caso pueden considerarse desde un punto de vista estructural grandes precarios. Por el contrario, lo que les define es formar parte de 'la media', de 'la clase media', de 'los sectores medios' de la sociedad, es decir, de aquellos que hasta hace poco se consideraban protegidos, tanto por sus recursos y competencias sociales como por los recursos destinados a ellos por parte del Estado de bienestar.

Sin contar los efectos de la reciente crisis financiera, estos sectores que ocupaban -y ocupan todavía- un lugar central en la sociedad española, han visto reducida su capacidad 
de movilización de recursos como resultado tanto de la erosión de las políticas públicas llevadas a cabo en la última década como del deterioro de los medios propios.

La metodología utilizada se orienta a la interpretación de los datos obtenidos mediante técnicas de investigación cualitativas, técnicas que profundizan en el sentido y significados que las personas entrevistadas dan a sus experiencias². Para el análisis de las definiciones y vivencias de la precariedad, las metáforas que utilizan para narrar su situación y las estrategias biográficas de los jóvenes ${ }^{3}$, se han realizado 12 entrevistas semi-estructuradas de carácter personal y 1 grupo de discusión ${ }^{4}$.

Tabla 1. Características de los participantes en las entrevistas individuales y grupo de discusión

E1 Mujer, 29 años. Licenciada en Magisterio. Trabajando de dependienta. Viviendo en pareja en piso con hipoteca.

E2 Mujer, 29 años. Licenciada en Derecho. Preparando oposiciones. Vive con sus progenitores.

E3 Hombre, 29 años. Graduado en Administración de Empresas. En paro. Vive con sus progenitores.

E4 Hombre, 29 años. Formación Profesional. Técnico medioambiental. En piso compartido y de alquiler.

E5 Hombre, 25 años. Estudiante. Empleos temporales. Viviendo en pareja en un piso de alquiler.

E6 Mujer, 32 años. Licenciada en Traducción e Interpretación. Traductora por cuenta propia. Viviendo en pareja en un piso de alquiler.

E7 Hombre, 29 años. Formación Profesional. Contratado temporal como estibador. Viviendo en pareja en piso con hipoteca.

E8 Hombre, 28 años. Ingeniero de Caminos, Canales y Puertos. Contratado temporal en construcción de infraestructuras. Jornadas de trabajo muy largas. Viviendo en pareja en un piso de alquiler.

E9 Mujer, 30 años. Diplomada en Educación Social. Empleos temporales. Con problemas de salud. En piso compartido y de alquiler.

E10 Hombre, 33 años. Con empleo estable. Vive con su madre.

E11 Mujer, 30 años. Madre soltera. Recibe la Renta de Garantía de Ingresos (RGI). Vive en vivienda de alquiler protegida.

E12 Mujer colombiana, 30 años. Madre soltera. Trabajos sin contrato. Vive de alquiler.

Grupo de 5 mujeres, en torno a los 30 años, emancipadas y no-emancipadas. Nivel de cualificación G1 medio-alto.

Fuente: Elaboración propia.

En el apartado siguiente se presentan los distintos significados y narrativas sobre las experiencias de precariedad vividas por las personas jóvenes entrevistadas.

2 Se puede encontrar un desarrollo cuantitativo del concepto de precariedad compleja en otros trabajos desarrollados a partir de la construcción de índices de precariedad (Tejerina y Llopis, 2015; Llopis y Tejerina, 2016). Estos índices atienden a distintas dimensiones de la precariedad de la población española, su comparación a lo largo del tiempo y entre distintos grupos de edad a partir de los datos proporcionados por la Encuesta de Condiciones de Vida del Instituto Nacional de Estadística.

3 El concepto de juventud y sus límites ha estado sujeto a numerosas discusiones. El más frecuente, y que concita mayor consenso, es el que cuestiona la homogeneidad de este agregado de edad. En el texto preferimos utilizar el genérico 'la juventud' en lugar de 'los jóvenes y las jóvenes'. Está fuera de lugar una utilización e interpretación hipostatizadora o reificadora del concepto de juventud por nuestra parte. Existe un acuerdo generalizado entre la mayoría de los estudiosos de la juventud de su configuración plural, de la diversidad y heterogeneidad de sus manifestaciones, así como de sus móviles límites dependiendo de épocas temporales, grupos étnicos y culturales, y realidades geográficas y espaciales. La totalidad de los jóvenes que han participado en entrevistas y reuniones de grupo responden a una extracción social de clase media o trabajadora, que residen en zonas preferentemente urbanas, con estudios profesionales medios o de nivel universitario, entre 20 y 34 años. Únicamente en dos casos se puede considerar que son muy precarios, es decir, afectados por dos dimensiones de la precariedad.

4 La información utilizada proviene de la transcripción de las entrevistas individuales y del grupo de discusión. Las características más relevantes de las personas jóvenes participantes figuran en la Tabla 1. 


\section{SIGNIFICADOS, NARRATIVAS Y EXPERIENCIAS DE LA PRECARIEDAD}

Hay un acuerdo bastante generalizado entre los científicos sociales sobre el diagnóstico de la importancia del riesgo y la incertidumbre en las sociedades contemporáneas (Bourdieu, 1999; Beck, 2000). La idea central de estos diagnósticos es que nos encontramos en una sociedad que ya no se caracteriza por la administración del riesgo sino por una incertidumbre radical.

La pregunta que queremos responder en este apartado es si esa incertidumbre general forma parte de la definición de la situación hoy, y si ha pasado a formar parte de la experiencia cotidiana de las personas jóvenes. Para ello utilizaremos los verbatims procedentes de entrevistas realizadas con jóvenes que, en general, no sufren grandes situaciones de precariedad, tienen un nivel de estudios medios o altos, y, en la mayoría de los casos, pueden disponer de alguna ayuda familiar o pública.

El uso del término precariedad en el lenguaje coloquial aparece frecuentemente asociado a la inestabilidad laboral. Si tomamos en consideración los testimonios recogidos, las personas entrevistadas tienden a identificar su posición en una escala que va de la estabilidad, asociada a un empleo ocupado durante muchos años o a un puesto de trabajo como empleado público, hasta la inestabilidad, descrita como una sucesión de empleos temporales o entradas y salidas constantes del mercado de trabajo. La inestabilidad tiende a asociarse con la falta de continuidad o duración en el tiempo:

Me salen cosas pero no me salen... Cosas temporales sí que me salen, o sea, pero programas concretos de quince días o de tal. Pues ahora me han llamado, esta mañana me han llamado del INEM para ir a [XXX]. Pero ahora, en julio, voy a trabajar también con el ayuntamiento, (...) ahí por las mañanas de julio (E5), o

Siempre he tenido trabajos, pero no he tenido un trabajo estable. ¿Por qué? Pues bueno, (...) después de la carrera me enfoqué a un tema de oposiciones y tal, que es muy difícil de encontrar, y además porque ahora el hecho de buscar trabajo es muy mala época (...). Ahora ando con un tema de rentas y todo esto del IRPF, y eso al final es una cosa que llevo haciendo varios años, pero que tiene la condición de que son tres meses porque está con un tema de hacienda, de información y tal. Son tres meses, y al final he trabajado con gente que está en la misma situación que tú, y son trabajos temporales (E2).

Una parte importante de la juventud se enfrenta al reto de alcanzar estabilidad en el empleo, especialmente en un momento de flexibilización del mercado de trabajo, con una oferta de sucesivos empleos precarios, malas condiciones de trabajo, y contratos de corta duración. En esta coyuntura, la idea de perder el trabajo está muy presente y resulta una vivencia cotidiana, con una rotación muy elevada de contratos de duración limitada a periodos cortos, de reducción de puestos de trabajo o de alta rotatividad, donde piensas "cualquier día soy yo" (Santamaría, 2011, p. 108).

Así, desde una postura pragmática, la máxima aspiración de algunos jóvenes es construir una vida basada en una estabilidad inestable.

La precariedad se declina, en segundo lugar, como una experiencia de desencaje (Urraco, 2017): no estoy en el lugar que debería, he perdido mi tiempo y se están desaprovechando las capacidades que he adquirido. Los siguientes verbatims reflejan claramente esta situación de desencaje, de no estar ubicado correctamente, de ocupar un lugar que no es -consideran los entrevistados- el que les corresponde:

Es que luego acabé el instituto, lo acabé muy bien. Claro, no era lo mismo el instituto que mi colegio. Y entonces, bueno, pues tiré por la universidad, quizás por darle el gusto a mis padres. Me gustaba, (...) pero me siento como que he perdido cinco años de mi vida. Siete, que tardé siete. Espero que no sea pérdida, pero fíjate como estoy. Digo yo que no serán pérdida, pero ahora, actualmente, lo siento como si fueran. Yo igual si hubiese ido a un módulo, pues 
hubiese estado dos años, y me hubiese quedado para trabajar, y olvídate ya de cuarenta códigos que no sirven para nada, que sirven, pero a mí no me sirven para nada. Pero bueno, lo hecho, hecho está, pues ya no me voy a cambiar (E3); y

[tengo] clarísimo que yo he estado estudiando pues para el día de mañana estar en un puesto de trabajo, a ver... buscando unas condiciones, ¿no?, pero con un horario distinto, con un trabajo completamente distinto, yo siempre quise trabajar con niños, y la educación es completamente distinto a lo que hago. (...) Quiero terminar de tienda, iquiero tener un trabajo para lo que he estudiado toda la vida! Entonces, por un lado, bien, pero, por otro, te agobias mucho (E1).

La vivencia de la precariedad se va subjetivando en un proceso de interiorización de la situación y de las respuestas para salir o evitar la falta de estabilidad. Según el testimonio de los jóvenes entrevistados, esto se consigue de dos maneras. En primer lugar, mediante la actualización permanente de los conocimientos y competencias adquiridas en la etapa formativa, que se prolonga a lo largo de la vida productiva:

Hoy en día creo que la formación es un oficio, o por lo menos es lo que... Porque antes no (...). 0 sea, es que el tema es muy... está bastante complicado. Y no puedes estar viviendo de lo que has estudiado hace cuatro o cinco años, porque eso ya se ha quedado obsoleto (E3).

En segundo lugar, echando mano de la capacidad emprendedora, de una inversión en las propias capacidades:

Igual hay gente arriesgada y toma la decisión y dice: 'Es que hay que hacerlo'. Y yo pienso que al final, y si eres valiente, yo creo que hay de todo, y que de todo se sale si eres una persona emprendedora (E7); y

Si tengo más tiempo hago formación, ¿por qué? Porque asi seré más competitiva y conseguiré poner lo que esté en mis manos para solventar esta crisis (G1).

Beck (2000) denomina a estos sujetos proletarios de la autorrealización, Foucault (2007) utiliza el término empresario de sí, y Lorey (2009) se refiere a la precarización de sí, para definir la situación de los productores culturales (Carbajo, 2016, p. 1).

La experiencia de la dificultad insalvable empuja a muchos jóvenes a buscar ayuda o colaboración de otros actores, principalmente familiares (Moreno, 2000) o procedentes del sector público (Aparicio y Crespo, 2017; Echaves, 2017). La mayor parte de los testimonios señalan la dificultad de salir adelante, de poder vivir con suficiencia:

Tengo que apretarme el cinturón un poco, porque con el sueldo y la ayuda pública no es suficiente (E12).

Son situaciones que pueden conducir a la desactivación de la persona, a tener la sensación de estar sobrepasada e impotente:

Me siento mal, impotente, de que no puedo hacer nada, porque está el niño ahí, y claro, y el niño es lo más importante de esta vida, ¿no? Y no lo voy a dejar abandonado (E11);

Si eres un poco cerrado, eres un poco introvertido, pues el tema es que igual este trabajo te puede comer, porque estás en la calle, eres carne de cañón (E10).

En las narrativas sobre la vida cotidiana de los jóvenes encontramos constantes referencias a los tópicos de la buena suerte y del infortunio «he tenido suerte, ya que estoy bien donde estoy» (E10). También cuando se refieren a la posibilidad de tener autonomía residencial mediante la compra de una vivienda, lo que únicamente parece suceder si tienes suerte o te toca una vivienda de protección oficial (VPO) por sorteo:

Cada vez es más difícil encontrar trabajo al nivel económico que te marca la vida, y los pisos

cada vez están más caros. Entonces para una persona sola es muy difícil si no tienes un sueldo 
bastante alto. Es muy difícil comprarte una casa si no tienes la suerte que te haya tocado una VPO o algo así (E4) ${ }^{5}$.

Cabe pensar que la referencia a los tópicos del infortunio, a la buena o mala suerte, no debe estar presente en el imaginario de las generaciones más jóvenes. Una visión pragmática de la existencia, racionalizada mediante la educación y los valores que predominan en la sociedad actual, conduce a una creciente secularización de la vida y de las prioridades vitales. Sin embargo, el tipo de testimonios que acabamos de reproducir nos recuerda que también la racionalización tiene límites, y que la resacralización de la existencia -o sacralización de lo profano- en términos de suerte, ventura, fortuna, azar, estrella o casualidad ocupa un lugar relevante en la atribución de dirección a nuestro destino.

Además de los medios personales disponibles, los recursos para paliar la precariedad vienen de dos fuentes principales: la familia y los subsidios públicos (Carbajo, 2014). La primera goza de una amplia aceptación y está generalizada entre los jóvenes que no viven lejos de la casa de sus parientes o les visitan periódicamente: «cuando visitamos a la familia regresamos con un tupper» (E4), e «intento que mi familia me ayude económicamente» (E6). La segunda está fuertemente estigmatizada, hasta el punto de que recibir estas ayudas es como vivir con muletas, convertirse en un sujeto protesizado, que no puede subsistir sin prótesis:

no me gusta tener que marcar una cita con la trabajadora social, no me gusta coger esto [ayuda social] (E12), y

No me gusta recibir esto [ayuda] ... Pues da pena y da como angustia. Luego ves a toda la gente que se nota que no tienen dinero, y dices 'jo' (...) entonces eso a la larga va cansando (E11).

Las definiciones de qué es precariedad están sujetas a variaciones en función de la posición social de los jóvenes entrevistados; pero, al mismo tiempo, las experiencias narradas tienden a marcar un territorio común, conocido y visitado frecuentemente o permanentemente, y un tiempo presente delimitado por la incertidumbre que hace difícil pensar el futuro (Artegui, 2017).

La normalidad implica una ausencia de incertidumbre, una cierta seguridad que garantiza unas condiciones de vida mínimas. Condiciones que pueden asegurarse a través de un empleo o de la percepción de una renta. Así lo explica este joven:

He tenido suerte, porque de alguna forma creo que tengo un colchón, un colchón que me permite tener casi, casi, y digo entre comillas 'casi', pero vamos, en última instancia asegurado un sueldo. Y mal que vengan las cosas tengo ese... No tengo ante mí como un vacío que diga: 'Dios mío, mañana qué va a ocurrir'. Creo que puedo decir que mañana voy a poder comer y que mis necesidades inmediatas están cubiertas (E9).

Resulta sorprendente constatar que las respuestas sociales de la juventud a estas situaciones de precariedad se declinan en singular, en términos individuales y del círculo de relaciones más próximo e íntimo, y, como señalan Muñoz y Santos (2014) las respuestas colectivas a la precariedad son minoritarias.

La gestión de estas situaciones de precariedad produce tres efectos relevantes sociológicamente. El primero tiene que ver con la significación tradicional del trabajo y sus resignificaciones sociales, mediante las que trabajo y no trabajo se contaminan mutuamente (Santamaría, 2011, p. 257). El segundo remite a una experiencia del tiempo donde la temporalidad se estira como momento de espera, de actividad de búsqueda, de ensayo y error, de experimentación que prolonga indeterminadamente la frontera simbólica de la edad adulta. El tercero produce trayectorias biográficas en permanente

5 Carbajo (2015) y Echaves (2018) han investigado intensamente sobre las relaciones entre vivienda, juventud y emancipación. 
reconfiguración, momentos de reinterpretación entre lo vivido y lo proyectado previamente, de identidades en precario. Las significaciones de la precariedad nos hablan de un territorio habitado y transitado por la juventud contemporánea, del que -en la mayoría de los casos- se desea escapar. En ese territorio se concitan actores principales, procesos estructurales, experiencias cotidianas, despliegue y gestión de capacidades y recursos, procesos de desactivación, ayudas, apoyos y colaboraciones, intervenciones privadas y públicas, e incluso entidades trascendentes. En el siguiente apartado nos detendremos sobre la manera de expresar estas situaciones a través de metáforas.

\section{METÁFORAS SOBRE LA PRECARIEDAD}

Donde no es posible llegar con el significado de las palabras del lenguaje común se puede llegar con la metáfora, figura retórica que ocupa el lugar de lo representado y ayuda a hacer visible algún aspecto esquivo. Tanto las entrevistas individuales como el grupo de discusión realizados están plagados de estas referencias secundarias, que con una gran economía de lenguaje nos aproximan los significados que la precariedad adopta, las consecuencias que tiene o las situaciones que produce. Estos elementos se presentan a través de seis metáforas e imágenes de cómo se vive la precariedad: vivir al día, estar en la cuerda floja, estrés a lo bestia, stand-by, un paso atrás y quiero ser normal.

La ausencia de estabilidad en los ingresos a largo plazo de los jóvenes adultos es fuente de desasosiego que cada persona gestiona de diferente manera. La carencia de estabilidad es la fuente principal de incertidumbre. La expresión con la que se alude a esta situación es suficientemente gráfica 'vivir al día'. Detengámonos en dos testimonios:

E4: 0 sea, no tengo casi dinero en el banco pero diariamente puedo vivir bien, más o menos bien, más o menos bien, pero no tengo casi dinero ahorrado.

Ent.: O sea, no da para ahorrar pero te da para mantenerte.

E4: Aunque después de tanto tiempo trabajando no tengo para ahorrar, pero bueno, también porque gasto. En verano me voy de vacaciones, y cosas así, entonces... (E4), y

Justito, hay meses que vivo mejor, meses que vivo peor (...) vivo un poco a salto de mata (E6).

Vivir al día, a la semana o al mes, vivir a salto de mata, pero sin un horizonte claro y despejado, forma parte de una etapa de transición, de redefinición y de ubicación, de búsqueda, pero también de ausencia de necesidad de planificar el futuro, de pensar básicamente en el presente, el presentismo (Muñoz, 2007).

Estas situaciones disparan el imaginario del riesgo entre la juventud. En especial, entre aquellas personas que son trabajadores autónomos, el término riesgo y sus derivados se articulan en torno al verbo arriesgar. Del análisis de un grupo de discusión realizado con mujeres jóvenes autónomas, Pérez-Agote y Santamaría (2008) concluyen que «arriesgan en el trabajo, arriesgan en la emancipación y arriesgan con la llegada de la descendencia» (pp. 141-142). Arriesgarse, atreverse, dar (y que te den) el empujón, tirarse a la piscina, lanzarse hacia delante, son otras formas de enunciar el vivir a la intemperie.

Una segunda manera de declinar la incertidumbre la encontramos en la expresión estar en la cuerda floja. La metáfora del equibrista, trapecista, o del slackline remite a situaciones de ausencia de anclajes seguros, de vaivenes que a modo de balanceo conducen a una existencia llena de oscilaciones. El testimonio de este joven lo expresa con suficiente claridad,

es estar un poco ahí, en el alambre, como los funambulistas, no sé. Algo precario es algo que no tiene mucho equilibrio, algo que... no sé, no sé cómo definirlo, que se puede venir abajo (E5). 
Estar en la cuerda floja prefigura un presente que puede hundirse en cualquier momento. Esta dimensión de vivir a salto de mata se ha acentuado entre los jóvenes como resultado de la crisis financiera y social de 2008 (Moreno, López y Segado, 2012; Alonso, Fernández e Ibañez, 2017), pero sus raíces son anteriores. Las dificultades se afrontan cuando aparecen, a medida que surgen, y una de sus primeras consecuencias es la imposibilidad o dificultad de elaborar proyectos a medio y largo plazo.

Esta forma de vivir configura una mentalidad y una serie de pautas para afrontar psicológicamente sus consecuencias. La experiencia de la precariedad tiene también implicaciones para el cuerpo al generar resiliencia por parte de los jóvenes para soportar situaciones difíciles y estresantes, pero también miedos y procesos de medicalización para convivir cotidianamente con ella. La ansiedad es un síndrome característico de las situaciones de precariedad. Detengámonos brevemente en ambas dimensiones. La falta de logro de las expectativas vitales, especialmente cuando no son realistas, refuerza la resistencia y la capacidad de gestionar estas situaciones:

Pues eso, la tolerancia a la frustración es bárbara, la gestión del estrés y de esa frustración, esa gestión, el aprovechar el momento, aprovechar el día, todo el rato, a lo bestia. Yo lo vivo así. Porque claro, igual no, o sí, esto puede ir para adelante. Yo, a ver, mi experiencia en estos años, es que voy yendo a peor... y a veces tienes la sensación de que sigues yendo a peor. Entonces claro, vamos a ver, tú no puedes quedarte en tu casa diciendo: 'a ver una medicina, a ver si encuentran una medicina, a ver si encuentran una medicina' (E9); y

he superado mis tendencias a engordar, pero no estoy contenta conmigo misma (E11).

En ciertos casos, estas situaciones se complican cuando van acompañadas de otras tareas de cuidado a familiares enfermos o dependientes, dando lugar a que la situación se vuelva incontrolable desde el punto de vista personal, donde la persona se siente sobrepasada, como señala esta joven,

Tendrán que apechugar mis hermanas también. Porque, claro, yo necesito desconectar. Necesitas desconectar ¿no? Porque vas ahí y entonces cuando está muy tonta [la persona dependiente], pues, muy bien, pero otros días dice 'no te vayas, quédate', entonces te agarra la mano. Y a mí me sacas hecha polvo, o sea, a mí me está matando. Sinceramente, me está matando. (...) Llega un momento en que he dicho 'no pienses en nada más que si hace buen tiempo y hace mal tiempo, porque yo creo que me voy a volver loca' (E12).

$\mathrm{Y}$, si se acude a la ayuda profesional, la medicalización de la ansiedad acrecienta el malestar y la desconfianza hacia el sistema de salud (E9).

La situación más común entre la juventud española es la experiencia de estar en un tiempo de espera, una situación en la que el desenlace de la trama tiene que producirse de un momento a otro, pero mientras llegan los buenos tiempos y las buenas noticias se posiciona en modo stand-by, conectado pero en reposo. Esta situación supone una prolongación, en ocasiones deseada, pero en general no querida, de la estancia en casa de los parientes, sin poder emanciparse y llevar una vida autónoma en solitario o en pareja. La carencia de un trabajo estable y la imposibilidad de vivir de forma independiente (Alguacil, 2017), prolongan la transición a la etapa adulta (Querol y Alcañiz, 2015), la fuga y salida (Santos y Muñoz, 2017) o el retorno a una vida dependiente (Gentile, 2010). E2 lo señala en la siguiente afirmación:

Yo sé ahora que tengo una edad... que tengo que desarrollarme ya, no puedo estar viviendo a costa de mis padres toda la vida, porque ya al final aprieta eso un poco. Me tengo que ir de casa. Bueno, a ver, nadie me echa de casa; pero, bueno, yo también quiero desarrollarme y tal. Y yo creo que actualmente, para poder irme de casa, yo necesito un trabajo estable. Así de claro.

También los proyectos en pareja se ven afectados por esta situación de estar a la espera, como apunta E8, 
Pues mira, llevamos, para que te des una idea, once años de pareja. 0 sea, prácticamente desde que empezamos todo para adelante. Entonces, cuando llevábamos siete u ocho años ya, como es lógico, ya te pica el gusanillo de tener tu propia vivienda;

$y$, en palabras de E3,

Tengo ganas ya, tengo ya treinta años y me apetece mucho, mucho. Tengo novia, llevo año y medio con ella, y me gustaría no irme a vivir con ella en cuanto tenga una vivienda, porque las cosas hay que tener hoy en día mucho cuidado, pero, bueno, sí que tengo ganas de intentarlo.

Estos testimonios representan un tipo de experiencia generalizada entre la juventud contemporánea, y no algo que afecta a una minoría, hasta el punto que conceptos como el de transición a la vida adulta o en qué consiste ser adulto están en cuestión, y requieren, al menos, una redefinición. Otros autores han ido más lejos, apuntando la necesidad de olvidarnos de los calificativos más espectaculares para prestar más atención a los patrones que los jóvenes 'normales' utilizan para resignificar tanto la juventud como la edad adulta (Woodman, 2013). Encontramos un ejemplo claro de esta crisis en el análisis de la etapa de transición de la formación al empleo. Los testimonios abundan en el desajuste entre formación y empleo ocupado. Se produce aquí un desencaje entre capacidades y oportunidades. La respuesta más frecuente es la de dar un paso atrás para poder dar un paso adelante, aparentemente la única manera de reensamblar las piezas desencajadas: "yo creo que tendría que haber estudiado otra cosa» (E2). En otras ocasiones, se vuelve a estudiar, en especial cuando se está sobrecualificado, para intentar adquirir una formación más acorde con lo que se desea o para incrementar las oportunidades de encontrar otro empleo, entrando en una espiral de recualificación, sobreeducación, sobrecualificación (Budría y Moro-Egido, 2008; García-Montalvo y Peiró, 2009; Nieto y Ramos, 2010).

La precariedad está tan presente en la sociedad contemporánea que hay muchos jóvenes que 'desean ser normales', lo que llevaría a preguntarnos ¿en qué consiste la normalidad? Querer ser normal, la normalidad se declina como ausencia de incertidumbre, identificándose con los actos, prácticas y deseos comunes y banales de la vida cotidiana: «me gustaría verme de padre de familia, con dos hijos, y llevando a mis hijos a ver al Athletic» (E3), o, simplemente,

Salir de casa y hacer mi vida... yo. Que luego sea con pareja, pues muy bien, oye, pero primero yo. Y luego viene todo lo demás. Ya sé que suena muy egoísta, pero primero yo, y luego todo lo demás. Primero salir yo, luego verás como viene (E2).

Una consecuencia clave de la presencia cotidiana de la precariedad y la ausencia de normalidad es la dificultad para imaginar el futuro hacia el que caminar desarrollando un proyecto. Como ha señalado A. Lasén (2000), la propia noción de proyecto ha cambiado de sentido, puesto que no remite a una trayectoria individual planificada sino a momentos desconectados en los que se mezcla lo real y vivido con lo virtual, los ensueños y fantasías (p. 116).

\section{CONCLUSIONES}

La relación de la juventud española con la precariedad a partir de mediados de la década de 1980 ha experimentado cambios importantes. La precariedad que, en un primer momento, era frecuente encontrar en sectores menos favorecidos de la sociedad se ha ido extendiendo a buena parte de las personas jóvenes. La crisis financiera que comenzó en 2008 ha profundizado las experiencias de falta de estabilidad en el empleo e irregularidad en los recursos económicos disponibles. Sin embargo, continúan existiendo diferencias significativas dentro de la juventud (Tejerina et al., 2012), y entre la juventud y otros grupos sociales (Llopis y Tejerina, 2016). La precariedad y la incertidumbre asociada a ella se han 
convertido en una experiencia común en la vida cotidiana de los contemporáneos. Es posible que, si tiene razón Inglehart, las personas que han sido socializados en su etapa juvenil en una determinada época de bonanza o escasez construyan visiones del mundo y valores sociales claramente diferenciados. Y en ello descansa una parte del interés sociológico por conocer y profundizar en los sentidos que la precariedad adopta entre la juventud española.

Los resultados del análisis de las estrategias biográficas de la juventud actual frente a la precariedad se presentan en torno a seis argumentos que articulan una visión complementaria a los avances de estudios previos sobre la misma cuestión.

Aunque existen diferencias importantes entre los distintos tipos de precariedad, así como grados menores o mayores de precariedad, en muchos casos encontramos que la juventud experimenta un desajuste entre sus capacidades y su plasmación concreta. Este gap empuja a dar un paso atrás para poder continuar avanzando: volver a estudiar, estudiar otra cosa, recualificarse, cambiar de lugar de residencia, reinventarse. La idea que se persigue con ello es reensamblar dos realidades que se perciben desencajadas. Esta experiencia del desencaje ha llevado a un número considerable de jóvenes a buscar alternativas en el extranjero o a tratar de reinventarse bajo otros paraguas. Si bien la precariedad se muestra bajo la hegemonía de la precariedad laboral, otras dimensiones como la formativa, residencial, relacional, han ido adquiriendo un protagonismo creciente.

La precariedad individual, en segundo lugar, no se percibe como algo que tiene un origen estructural, por lo que la gestión de estas situaciones se orienta a cambios individuales y procesos de transformación personal. Es cierto que, en algunos casos, las situaciones de precariedad llevan a una marcada hiperactividad, que no conviene confundir con sobremotivación pues, según ciertos testimonios, también desarma y desalienta a quien tiene que enfrentarlas. La escasa acción colectiva y movilización social no es sino un indicador más de esta individualización. En todo caso, una activación juvenil minoritaria, que ha protagonizado algunas de las grandes movilizaciones de la última década, resulta compatible con una mayoritaria apatía frente a la participación activa en política.

Socialmente, la responsabilidad de la situación es, en tercer lugar, casi siempre atribuida a cada persona, a quien se demanda una respuesta resiliente. Ello conduce a un trabajo sobre la propia identidad, a una constante actividad de invertir en uno mismo, lo que se ha denominado empresarización de sí. Individualmente, la precariedad se vive como un conjunto de situaciones negativas desde el punto de vista de la salud y el bienestar. Un aumento de los padecimientos físicos y, sobre todo, psíquicos entre los jóvenes puede guardar relación con esta precarización generalizada.

La solidaridad familiar, en cuarto lugar, es clave para entender cómo la juventud puede lograr estabilidad en situaciones de precariedad, así como la existencia de soportes sociales mediante ayudas o subsidios públicos. A la luz de los testimonios aportados por los jóvenes españoles, es razonable pensar que el bienestar de muchos de ellos se apoye más en las ayudas familiares y en menor medida, aunque existen grandes diferencias según zonas geográficas, en las facilitadas por el propio Estado y las Administraciones públicas. Pero mientras la ayuda familiar se acepta sin dificultad, la segunda puede ir acompañada, en algunos casos, de estigmatización social y personal.

La percepción temporal en la que vive instalada la juventud actual, en quinto lugar, hace que resulte casi imposible la planificación a medio y largo plazo. Vivir al día y conjugar la existencia en tiempo presente es el correlato de la banalización de la incertidumbre. La idea de precariedad laboral, residencial o vital tiene otra consecuencia importante: la dificultad de imaginar el futuro y el por-venir. No se trata tanto de la imposibilidad de imaginar o delinear el futuro como de los límites de los futuros proyectados. 
Finalmente, el tránsito de la dependencia a la independencia que antes ocupaba un periodo de tiempo corto se ha prolongado en las últimas décadas. La prolongación de los años dedicados a la formación, así como las condiciones laborales actuales y las dificultades de estabilizarse en el empleo, dilatan el tiempo utilizado para realizar esta transición. El resultado es que aparecen nuevas figuras juveniles y la propia categoría de adulto se desdibuja, al no establecer ya, en muchos casos, una ruptura clara con etapas anteriores como sucedía previamente. Lejos de mantenerse a la espera durante esta juventud prolongada, lo que habitualmente encontramos es una constante actividad para soportar o superar los impactos de la precariedad.

Como ha señalado Santamaría (2011), estos futuros comparten cuatro rasgos: la indeterminación, expectativas a corto plazo, el carácter azaroso de la construcción biográfica y la construcción de metas vitales poco pretenciosas (p. 294). Esperar, ver qué pasa, actuar si no pasa nada, no pensar e idealizar el mañana, así construyen muchos jóvenes el futuro, haciendo diariamente lo que está a su alcance, con los repertorios disponibles a la mano.

\section{AGRADECIMIENTOS}

Este artículo ha contado con el apoyo económico del proyecto "Sharing society. El impacto de la acción colectiva colaborativa" financiado por el Ministerio de Economía y Empresa del Gobierno de España CSO2016-78107-R, así como del Grupo de investigación consolidado del Sistema Universitario Vasco IT1199-19. Quiero agradecer la colaboración y las contribuciones de Diego Carbajo, Beatriz Cavia, María Martínez y Elsa Santamaría en la investigación, así como los comentarios de los evaluadores anónimos de este artículo. Este artículo se ha llevado a cabo en el marco de la Red de Excelencia sobre Juventud y Sociedad (REJS 2.0). Ministerio de Ciencia, Innovación y Universidades. CSO2017-90618-REDT.

\section{REFERENCIAS BIBLIOGRÁFICAS}

Alguacil, A. (2017). Jóvenes buscan piso: la distopía del acceso a la vivienda. Revista de Estudios de Juventud, (116), 125-142.

Alonso, L. E. (2000). Trabajo y postmodernidad: el empleo débil. Madrid: Fundamentos.

Alonso, L. E., Fernández, C. J. e Ibañez, R. (2017). Juventud y percepciones de la crisis: precarización laboral, clases medias y nueva política. Empiria: Revista de Metodología de Ciencias Sociales, (37), 155-178. https://doi.org/10.5944/empiria.37.2017.18983

Ancelovici, M., Dufour, P. y Nez, H. (eds.). (2016). Street Politics in the Age of Austerity. Amsterdam: Amsterdam University Press.

Aparicio, A. y Crespo, E. (2017). Fomento de la emancipación de los jóvenes: evidencias sobre el impacto del subsidio de alquiler español. Revista de Estudios de Juventud, (116), 43-48.

Ariño, A. (2004). Asociacionismo, ciudadanía y bienestar social. Papers: Revista de Sociología, 74, 85-110. http://dx.doi.org/10.5565/rev/papers/v74n0.1088

Artegui, I. (2017). El impacto de la incertidumbre en la transición a la edad adulta: posiciones biográficas y consecuencias sobre la vida. Arbor: Ciencia, Pensamiento y Cultura, 193(784), a379. https://doi.org/10.3989/arbor.2017.784n2002

Beck, U. (2000). Un nuevo mundo feliz. La precarización del trabajo en la era de la globalización. Barcelona: Paidós. 
Bilbao, A. (1998). El trabajador precario. Arxius de Sociología, 2, 39-56.

Bourdieu, P. (1999). Actualmente la precariedad está en todas partes. En Contrafuegos (pp. 120-128). Barcelona: Anagrama.

Budría, S. y Moro-Egido, A. (2008). Education, Educational Mismatch and Wage Inequality: Evidence for Spain. Economics of Education Review, 27(3), 332-341. https://doi.org/10.1016/j.econedurev.2006.10.005

Carbajo, D. (2014). Vivir en la precariedad. Trayectorias y estrategias residenciales de la juventud en la Comunidad Autónoma del País Vasco (Tesis de doctorado). Universidad del País Vasco, Departamento de Sociología II, Leioa.

Carbajo, D. (2015). Los procesos de precarización de la juventud en la CAPV a través de sus trayectorias residenciales. Vitoria-Gasteiz: Servicio Central de Publicaciones del Gobierno Vasco.

Carbajo, D. (2016). Proletarios de la auto-realización: un abordaje crítico del emprendimiento juvenil. Trabajo presentado en XII Congreso de la Federación Española de Sociología, Gijón.

Carrasquer, P. y Torns, T. (2007). Cultura de la precariedad: conceptualización, pautas y dimensiones. Una aproximación desde la perspectiva de género. Sociedad y Utopía, (29), 139-156.

Casal, J. (1996). Modos emergentes de transición a la vida adulta en el umbral del siglo XXI: aproximación sucesiva, precariedad y desestructuración. Revista Española de Investigaciones Sociológicas, 75, 296-316.

Casal, J., García, M., Merino, R. y Quesada, M. (2006). Cambios en las modalidades de transición en los países de capitalismo informal. Papers: Revista de Sociología, (79), 195-233.

Castel, R. (1997). La metamorfosis de la cuestión social. Una crónica del salariado. Buenos Aires: Paidós.

Dahrendorf, R. (1990). El conflicto social moderno: ensayo sobre la política de la libertad. Madrid: Mondadori.

Della Porta, D. (2015). Social Movements in Times of Austerity: Bringing Capitalism Back into Protest Analysis. Cambridge: Polity Press.

Díaz-Salazar, R. (ed.). (2003). Trabajadores precarios. El proletariado del siglo XXI. Madrid: Hoac, D. L.

Echaves, A. (2017). Emancipación residencial y sistema de provisión de vivienda: la heterogeneidad autonómica del modelo español. Revista Española de Investigaciones Sociológicas, 159, 51-72. http://dx.doi.org/10.5477/cis/reis.159.51

Echaves, A. (2018). Emancipación residencial y sistema de provisión de vivienda en España: hacia un análisis explicativo comparado por comunidades autónomas. Madrid: Politeya.

Foucault, M. (2007). Nacimiento de la biopolítica: curso en el Collège de France (1978-1979). México: Fondo de Cultura Económica.

García-Montalvo, J. y Peiró, J. M. (2009). Análisis de la sobrecualificación y la flexibilidad laboral. Valencia: Fundación Bancaja/IVIE.

Gentile, A. (2010). De vuelta al nido en tiempo de crisis. Los boomerang kids españoles. Revista de Estudios de Juventud, (90), 181-203. 
Giddens, A. (1984). The Constitution of Society: Outline of the Theory of Structuration. Cambridge: Polity.

González, M., Jiménez, I. y Morgado, B. (2004). Los retos de la maternidad en solitario. Revista de Estudios de Juventud, (67), 145-163.

Izaola, A. (2017). Miradas cruzadas. La construcción social de la otredad. Barcelona: Bellaterra.

Jiménez, B., Martín, Á., Navarrete, J., Pinta, P., Soler, R. y Tapia, Á. (2008). La emancipación precaria: transiciones juveniles a la vida adulta en España a comienzos del siglo XXI. Madrid: CIS.

Jurado, T. (2007). La precariedad temporal-salarial y sus efectos sobre la formación familiar. Sociedad y Utopía, (29), 367-404.

Lasén, A. (2000). A contratiempo. Un estudio de las temporalidades juveniles. Madrid: CIS/ Siglo XXI.

Le Blanc, G. (2007). Vidas ordinarias, vidas precarias. Buenos Aires: Nueva Visión.

Llopis, R. y Tejerina, B. (2016). Crisis, educación y precariedad-afluencia. El rol de la educación en las condiciones de vida de la población española. Política y Sociedad, 53(2), 413-442. https://doi.org/10.5209/rev_POSO.2016.v53.n2.49370

López, A. (2005). Excluidos pero trabajadores: el círculo vicioso de los 'trabajadores con bajo salario' en España. Cuadernos de Relaciones Laborales, 23(1), 153-174.

Lorey, I. (2009). Gubernamentalidad y precarización de sí. Recuperado de http://eipcp.net/transversal/1106/lorey/es

Mendiguren, A., García, J. y Ruiz-Estramil, I. B. (2017). El gobierno contemporáneo de la exclusión social extrema en Bilbao: dispositivos integradores y procesos de autorresponsabilización. Cuadernos de Relaciones Laborales, 36(1), 125-145.

https://doi.org/10.5209/CRLA.59560

Montero, R., Font, J. y Torcal, M. (2006). Ciudadanos, asociaciones y participación política en España. Madrid: CIS.

Moreno, A. (2000). Vida familiar y trabajo en el proceso de transición a la vida adulta de los jóvenes españoles en perspectiva comparada. Revista de Estudios de Juventud, (90), 123-141.

Moreno, A., López, A. y Segado, S. (2012). La transición de los jóvenes a la vida adulta. Crisis económica y emancipación tardía. Barcelona: Obra Social La Caixa.

Morente, F. y Barroso, I. (2003). La precariedad familiar ante la pobreza de la infancia. Una aproximación sociológica. Portuaria. Revista de Trabajo Social, 3, 67-88.

Mosca, L. (2006). May Day parade. Movilizaciones juveniles contra la precariedad laboral. Revista de Estudios de Juventud, (75), 75-97.

Muñoz, A. (2007). Tácticas de comunicación juvenil: intervenciones estéticas. Revista de Estudios de Juventud, (78), 11-23.

Muñoz, D. y Santos, J. A. (2014). Hoy es el futuro. De la activación universitaria a las respuestas colectivas frente a la precariedad juvenil. Revista de la Asociación de Sociología de la Educación, 7(3), 658-673. 
Nieto, S. y Ramos, R. (2010). Sobreeducación, educación no formal y salarios: evidencia para España. Documentos de Trabajo FUNCAS, (577). Recuperado de https://www.funcas.ceca.es/publicaciones_new/

Parés, M. y Subirats, J. (2016). Muy jóvenes, jóvenes y menos jóvenes: el lío de la juventud y la política. Revista de Estudios de Juventud, (114), 45-58.

Paugam, S. (1991). La disqualification sociale. Essai sur la nouvelle pauvreté. Paris: Puf.

Paugam, S. (2000). Le salarié de la précarité. Les nouvelles formes de l'intégration professionnelle. Paris: Puf.

Paugam, S. (2007). Las formas elementales de la pobreza. Madrid: Alianza Editorial.

Pereda, C. (2002). Plataforma de debate contra el paro y la precariedad. Una experiencia de trabajo en red. Documentación social, 129, 257-271.

Pérez-Agote, A. y Santamaría, E. (2008). Emancipación y precariedad en la juventud vasca. Entre la anomia funcional y el cambio cultural. Vitoria-Gasteiz: Servicio Central de Publicaciones del Gobierno Vasco.

Pitrou, A. (1978). La vie précaire. Les familles face à leurs difficultés. París: Études CNAF.

Portos, M. y Masullo, J. (2017). Voicing Outrage Unevenly: Democratic Dissatisfaction, Nonparticipation, and Participation Frequency in the 15-M Campaign. Mobilization: An International Quarterly, 22(2), 201-222. https://doi.org/10.17813/1086-671X-22-2-201

Prieto, C. (2008). Del estudio del empleo como norma social al de la sociedad como orden social. Papeles del CEIC, (1).

Prieto, C., Ramos, R. y Callejo, M. J. (2008). Nuevos tiempos del trabajo: entre la flexibilidad competitiva de las empresas y las relaciones de género. Madrid: CIS.

Querol, V. y Alcañiz, M. (2015). Jóvenes y trayectorias a la vida adulta: desigualdades, retos y nuevas formas en un contexto de crisis. Recerca: Revista de Pensament i Analisi, (16), 7-12. https://doi.org/10.6035/Recerca.2015.16.1

Rico, N. (2005). Jóvenes: precariedad más allá de la temporalidad y respuestas sindicales para construir el futuro. Gaceta Sindical. Reflexión y Debate, 5, 285-295.

Rodríguez, J. M. (1999). La sorpresa no era la emancipación adulta: autonomía virtual y dependencia real en la juventud española de la década de los noventa. Revista de Estudios de Juventud, (45), 103-111.

Salido, O. y Martín, A. (2007). Las urnas de la precariedad: el anclaje sociolaboral del voto juvenil en el 14-M. Sociedad y Utopía, (29), 463-487.

Sánchez Moreno, E. (2004). Jóvenes: la nueva precariedad laboral, La experiencia de la precariedad laboral en los jóvenes españoles. Madrid: Confederación Sindical de Comisiones Obreras.

Santamaría, E. (2011). Trayectorias laborales en los márgenes del empleo: políticas, subjetividades y experiencias de jóvenes en la precariedad laboral. Vitoria-Gasteiz: Servicio Central de Publicaciones del Gobierno Vasco.

Santos, A. (2003). Jóvenes de larga duración: biografías laborales de los jóvenes españoles en la era de la flexibilidad informal. Revista Española de Sociología, (3), 87-98.

Santos, J. A. y Muñoz, D. (2017). La subjetivación del capital humano: la movilidad como inversión en los discursos de los jóvenes universitarios que han protagonizado la fuga de cerebros. Areas: Revista Internacional de Ciencias Sociales, (36), 127-139. 
Schnapper, D. y Villac, M. (1989). Rapport à l'emploi, protection sociale et status sociaux. Revue Française de Sociologie, 30(1), 3-29.

Schütz, A. y Luckmann, T. (2001). Las estructuras del mundo de la vida. Buenos Aires: Amorrortu.

Sennett, R. (2000). La corrosión del carácter. Las consecuencias personales del trabajo en el nuevo capitalismo. Barcelona: Anagrama.

Standing, G. (2011). The Precariat. The New Dangerous Class. Londres: Bloomsbury Academic.

Subirats, J. y Gallego, R. (2002). Veinte años de autonomías en España: leyes, políticas públicas, instituciones y opinión pública. Madrid: CIS.

Tejerina, B., Cavia, B., Santamaría, E. y Carbajo, D. (2012). Precariedad vital y juventud vasca. Condiciones sociales y estrategias biográficas para llevar una vida normal. VitoriaGasteiz: Servicio Central de Publicaciones del Gobierno Vasco.

Tejerina, B. y Llopis, R. (2015). Capital educativo y precariedad: cambios recientes en las condiciones de vida en España (1995-2010). Oñati Socio-legal Series, 5(4), 1050-1085.

Tejerina, B. y Perugorría, I. (2018). Crisis and Social Mobilization in Contemporary Spain. The 15M Movement. Londres: Routledge.

Tejerina, B. y Seguel, A. (2011). Précarité et action collective dans la mobilisation altermondialiste. Réinterprétation et resignification de la vie en précaire. En S. Fortino, B. Tejerina, B. Cavia y J. Calderon (eds.), Précarités et sociétés. Modes de vie, travail et résistances en France et en Espagne (pp. 263-281). París: Erès.

Tobío, C. y Fernández Cordón, J. A. (1999). Monoparentalidad, trabajo y familia. Revista Internacional de Sociología, 22, 67-97.

Touraine, A. (1978). La voix et le regard. París: Seuil.

Turner, V. (1988). El proceso ritual. Estructura y antiestructura. Madrid: Taurus.

Urraco, M. (2017). Un saco de niños 'zaleados': precariedad laboral y precariedad vital de la 'generación de la crisis' en Extremadura (Tesis de doctorado). Universidad Complutense de Madrid, Facultad de Ciencias Políticas y Sociología, Departamento de Psicología Social, Madrid.

Van Gennep, A. (1986). Los ritos de paso. Madrid: Taurus.

Woodman, D. (2013). Researching ‘Ordinary' Young People in a Changing World: The Sociology of Generations and the 'Missing Middle' in Youth Research. Sociological Research, 18(1), 1-12. https://doi.org/10.5153/sro.2868

Zubero, I. (2006). Flexibilización del trabajo y precarización vital: el reto de la cohesión social. Revista de Fomento Social, (244), 519-560. https://doi.org/10.32418/rfs.2006.244.2126

\section{NOTA BIOGRÁFICA}

Benjamín Tejerina es Catedrático de Sociología y Director del Centro de Estudios sobre la Identidad Colectiva de la Universidad del País Vasco. Entre sus publicaciones más recientes se encuentran Crisis and Social Mobilization in Contemporary Spain: The 15M Movement (editado con I. Perugorría, Routledge 2018); Pensar la agencia en la crisis (editado con G. Gatti, CIS 2016); La sociedad imaginada. Movimientos sociales y cambio cultural en España (Trotta 2010). 